\title{
Efecto de la Socialización Organizacional en el Engagement de Trabajadores Mexicanos: Claridad y Futuro
}

\section{Effect of Organizational Socialization on Mexican workers' engagement: Clarity and Future}

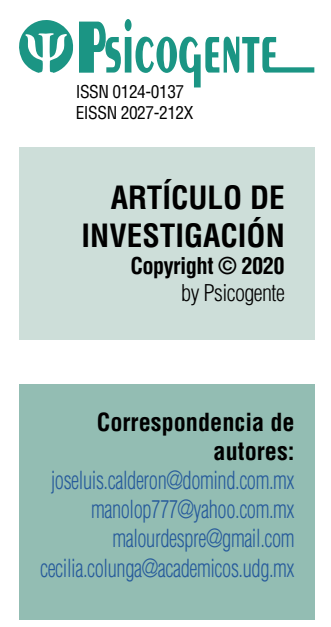

Recibido: 20/02/19 Aceptado: $13 / 08 / 19$ Publicado: 01/01/20

\author{
José Luis Calderón-Mafud iD \\ Universidad de Colima, Colima, México \\ Manuel Pando-Moreno (iD - Ma. de Lourdes Preciado-Serrano (iD) \\ Cecilia Colunga-Rodríguez (iD \\ Universidad de Guadalajara, Guadalajara, México
}

Resumen

Introducción: En el estudio del comportamiento organizacional se destaca con más frecuencia la importancia del proceso de socialización organizacional, relacionado con la adaptación al trabajo y el desarrollo de las competencias para el rol laboral; así como un interés creciente por los estados positivos en el trabajo como el work engagement (caracterizado por el vigor, dedicación y absorción) y que puede evidenciar la identificación del trabajador con la cultura de la organización.

Objetivo: Examinar el efecto de los dominios de la socialización organizacional sobre el work engagement que reportan tener los trabajadores mexicanos de la muestra.

Método: Estudio transversal-correlacional analítico, mediante análisis de regresión con los datos del Inventario de Socialización Organizacional de Taormina (1994), y el Utrecht Work Engagement Scale, en su versión al español por Schaufeli, Bakker y Salanova (2006), en 187 trabajadores mexicanos.

Resultados: Los análisis de regresión mostraron que la combinación de la comprensión funcional y las perspectivas de futuro se combinan para la aparición del vigor (Beta .29, .21), la absorción (Beta .23, .23) y la dedicación al trabajo (Beta .34, .25) en los trabajadores de la muestra. Se encontró que los cuatro factores de la socialización organizacional correlacionan con el vigor (.35), la absorción (.34) y la dedicación (.47).

Conclusiones: En los trabajadores de la muestra la comprensión y las perspectivas de beneficios, generan efectos en el vigor, la absorción y la dedicación al trabajo. Se señala la importancia de la socialización en la aparición de engagement como antecedente y componente de la productividad y el bienestar en el trabajo.

Palabras clave: socialización organizacional; work engagement; comportamiento en el trabajo.

Abstract

Introduction: not only the importance of the process of organizational socialization related to work adaptability and the development of competencies for the job role, has recently been highlighted more frequently in organizational behavior studies, but also a growing interest in positive states of work such as work engagement (characterized by vigor, dedication and absorption) and which can demonstrate the identification of the worker with the culture of the organization.

Objective: to examine the effect of the domains of organizational socialization on the work engagement reported by the Mexican workers in the sample.

Method: A transversal-correlational analytical study through a regression analysis with the data of the Inventory of Organizational Socialization of Taormina (1994), and the Utrecht Work Engagement Scale, in its Spanish version by Wilmar Schaufeli, Arnold Bakker and Salanova (2006), in 187 Mexican workers, was conducted.

Results: Regression analysis showed that the combination of understanding and future prospects were integrated for the display of vigor (Beta .29, .21), absorption (Beta .23, .23) and dedication to work (Beta .34, .25) in the sample workers. It was found that the four factors of organizational socialization correlate with vigor (.35), absorption (.34) and dedication (.47).dedication.

Conclusions: In the workers of the sample the understanding and the perspectives of benefits, generate effects in the vigor, the absorption and the dedication to the work. The importance of socialization in the appearance of engagement as a psychological state related to productivity and well-being at work is pointed out.

Keywords: organizational socialization; work engagement; behavior at work.

Cómo citar este artículo (APA):

Calderón-Mafud, J.L.; Pando-Moreno, M.; Preciado-Serrano, M. \& Colunga-Rodríguez, C. (2020). Efecto de la Socialización Organizacional en el Engagement de Trabajadores Mexicanos: Claridad y Futuro. Psicogente 23(43), 1-22. https://doi.org/10.17081/psico.23.43.3084 


\section{INTRODUCCIÓN}

En la actualidad los empleados de las diferentes organizaciones se enfrentan a retos como la incertidumbre financiera, implementación de nuevas tecnologías, escasez de mano de obra, problemas políticos e inseguridad pública, lo cual hace necesario que desarrollen la capacidad de adaptarse, avanzar y generar cambios positivos en las empresas que viven enormes presiones. Esto hace que el papel tradicional de la socialización organizacional, enfocado en adaptarse a una organización estática y sin cambios (Danielson, 2004), sea obsoleto y poco útil para afrontar las demandas del ambiente. Por otro lado, las necesidades constantes de socialización de individuos que viven cambios de empleo, de puesto de trabajo, tecnológicos o procesos de fusión, hacen necesario vincular la socialización con estados psicológicos positivos que permitan a las personas afrontar con éxito esos cambios (Rollag, Parise, \& Cross, 2005; Bauer, Bodner, Erdogan, Truxillo \& Tucker, 2007).

En ese contexto también es complicado para las organizaciones proponerse como objetivos la creación de culturas fuertes (Denison \& Mishra, 1995; Wallach, 1983). En vez de eso, promover una cultura organizacional orientada a la flexibilidad, basada en el apoyo social puede ser más apropiada para competir en los términos de la economía actual. La cultura organizacional se ha asociado con la eficacia organizacional, el comportamiento de liderazgo, la satisfacción laboral y con la atracción de empelados a las empresas (Macintosh \& Doherty, 2010; San Park \& Hyun Kim, 2009; Tsai, 2011; Gregory, Harris, Armenakis, \& Shook, 2009).

En la actualidad las personas están cada vez más interesadas en trabajar en empresas que poseen culturas organizacionales flexibles, en donde pueden encontrar mayores niveles de desarrollo y satisfacción laboral (Wallach, 1983; Lund, 2003; McKinnon, Harrison, Chow, \& Wu, 2003; Silverthorne, 2004).

La socialización organizacional, puede ser una evidencia de que existe adaptación a la organización y que este proceso es un facilitador del compromiso y el bienestar laboral. El modelo de Taormina $(1994 ; 1997)$ es uno de los varios enfoques que explica esta variable y está basado en cuatro dominios de contenido (Entrenamiento, Comprensión, Apoyo de los compañeros y Perspectivas de futuro), los cuales se desarrollan durante toda la vida laboral de forma simultánea, en diferentes grados (Wanous, 1992; Cooper-Thomas \& Anderson, 2006; Lisbona, Morales \& Palací, 2009; Chao, O’Leary-Kelly, Wolf, Klein \& Gardner, 1994); también se pueden entender como esferas de influencia o actividades que interactúan entre sí (Taormina, 
1994; 1997; 2004). Este modelo ha sido retomado por diversos autores como base para investigaciones recientes en donde se han identificado relaciones de los dominios de socialización con el compromiso afectivo y normativo hacia la organización (Calderón, Laca, Pando \& Pedroza, 2015). Igualmente, la socialización organizacional se ha visto vinculada con conductas de motivación y proactividad (Lisbona, et al., 2009) y también se han sugerido estudios empíricos con el capital psicológico y otras variables positivas como el engagement y el compromiso organizacional (Simons \& Buitendach, 2013; Luthans, Norman, Avolio \& Avey, 2008).

La mayoría de las investigaciones centradas en el estudio de las variables que influyen en el rendimiento, satisfacción y salud del personal en las organizaciones han sido realizadas desde una visión negativa. Sin embargo, en los últimos 20 años, el estudio de los estados psicológicos considerados como positivos ha surgido como contrapeso al enfoque patologizante de la psicología. Schaufeli, Salanova, Gonzáles-Romá y Bakker (2002) introdujeron el concepto de work engagement como el estado psicosocial positivo caracterizado por vigor, dedicación y absorción. Mientras que la socialización organizacional es un tema de investigación que se ha vinculado recientemente con la salud ocupacional y el bienestar.

La relación entre la socialización organizacional y el engagement ha sido resaltada por diversos autores (Lisbona, et al., 2009; Bamford, Wong \& Laschinger, 2013; Csikszentmihalyi, 1990; Konstantellou, 2001 \& Calderón et al., 2015), para los cuales la implicación de los conocimientos y capacidades en el trabajo, la realización de tareas de manera efectiva tanto individualmente como colectivamente, el planteamiento de metas personales encaminadas a las metas de la organización, el incremento de la productividad y la aceptación de la cultura organizacional, presentan una relación estrecha con fenómenos como el compromiso organizacional, la satisfacción laboral y la socialización organizacional.

\subsection{Socialización Organizacional}

Para un individuo que ingresa a un nuevo empleo, una nueva organización, o tiene un ascenso, esta etapa es considerada crítica (Wang, KammeyerMueller, Liu \& Li, 2015; Saks \& Gruman, 2018; Holton, 1996; Wanous, 1980). Durante este lapso, el empleado puede desarrollar habilidades para el trabajo y en general enfrentar las demandas del ambiente organizacional que requiere su nuevo rol. 
En muchas ocasiones, la socialización efectiva enfrenta la necesidad de superar aspectos negativos potenciales típicos de la adaptación a un nuevo empleo, tales como el estrés, la ansiedad e incertidumbre frente a la nueva situación y tareas (Louis, 1980; Nelson \& Quick, 1991; Wanous, 1992; Saks, 1996; Ellis, Bauer, Mansfield, Erdogan Truxillo \& Simon, 2015; Mafud, Arocena \& Moreno, 2017). En algunos casos los empleados deciden abandonar la organización al no lograr adaptarse a ella. Según diferentes investigaciones, hasta el $25 \%$ de los trabajadores mexicanos podrían hacerlo en los primeros 6 meses (Wanous, 1992; Chao, et al., 1994; Calderón Mafud, et al., 2015).

Este ajuste llamado socialización organizacional tiene diferentes aspectos que lo componen y que en general tiene que ver con cambios personales en el nuevo trabajador como efecto del cambio de los individuos con los que interactúa (Bauer \& Erdogan, 2014; Major, Kozlowski, Chao \& Gardner, 1995). Cuando una persona se adapta a la nueva cultura organizacional, también sus compañeros de trabajo, jefe y la organización reciben beneficios de ello porque existen relaciones de la socialización que favorecen la satisfacción laboral y el compromiso organizacional, además de los cambios positivos en la interacción social (Song, Chon, Ding \& Gu, 2015; Bauer, Morrison, Callister, \& Ferris, 1998; Cable \& Parsons, 2001; Fisher, 1986; Klein \& Weaver, 2000).

Los vertiginosos cambios tecnológicos y sociopolíticos que experimentan las organizaciones imponen al proceso de socialización una orientación hacia la constante innovación del sistema organizacional, y es posible que el aprendizaje de la cultura busque más la adaptación a los cambios culturales que únicamente mantener la cultura establecida y obstaculizar con ello la capacidad de una organización para ser ágil y adaptarse al medio ambiente (Danielson, 2004; Wanous, 1980; Chao et al., 1994; Taormina, 1994; 1997; 2004).

Aunque la mayor parte de la investigación sobre este tema se ha centrado en el proceso de aprendizaje o adaptación, algunos autores han enfocado sus estudios en los factores de interacción implicados en el éxito de este proceso (Taormina \& Gao, 2008; Jones, 1986; Hesketh \& Myors, 1997). Estos enfoques sostienen que es necesario prestar mayor atención a las necesidades de los empleados (Vandenberg \& Scarpello, 1994) y a la necesidad de la cultura organizacional (Schein, 1996), considerando a la socialización de organización desde una perspectiva interaccionista. En ella se sugiere que existe alguna influencia recíproca entre los individuos en transición y 
los miembros de mayor antigüedad, quienes facilitan la comprensión con sus conocimientos sobre la organización (Jones, 1986; Wanous, 1980; Allee, 1997; Amidon, 1997; Nonaka, Takeuchi \& Umemoto, 1996; Danielson, 2004).

Los componentes del modelo de Taormina son:

a. El entrenamiento, que se entiende como los actos, procesos o métodos mediante los cuales se adquieren habilidades funcionales o para realizar un trabajo específico (Louis, 1980; Feldman \& Brett, 1983). En este dominio se enfatiza la valoración que un empleado hace del nivel de experiencias que la organización le ha brindado y que le sirven para desarrollar las competencias consideradas adecuadas y suficientes para realizar exitosamente el trabajo (Taormina, 1997).

b. La comprensión funcional de la organización es "la medida en que un empleado comprende plenamente y puede aplicar conocimientos sobre su trabajo, la organización, su gente y su cultura" (Taormina, 1997, p.34). De este modo, comprensión se refiere a que el empleado logre comprender plenamente cómo funcionan las reglas y normas de la organización, su cultura, la forma de interactuar y su forma de operar.

c. El apoyo de los compañeros de trabajo se refiere al soporte emocional, moral o instrumental (que excluye las compensaciones financieras por parte de la empresa, jefes u otros empleados de la organización). Esta área se refiere a la cantidad de aceptación que un empleado logra obtener de sus compañeros como efecto de haber mostrado competencias y comportamientos apropiados (Taormina, 1994; 1997; Taormina \& Gao, 2008).

d. Perspectivas de futuro es la cantidad de beneficios que un empleado prevé que podría llegar a tener en su carrera laboral dentro de la empresa en que trabaja. Este dominio podría verse representado por recompensas económicas, bonos y las posibilidades de ascenso que uno percibe tener en una organización.

Taormina y Gao (2008) encuentran compatibilidad y coherencia entre su propuesta y otros dos modelos teóricos de socialización organizacional (Chao et al., 1994; Cooper-Thomas \& Anderson, 2006; Taormina, 1994; 1997), que estudian el contenido de la socialización, las áreas y el proceso respectivamente. El enfoque de Taormina (1997) estaba compuesto por cuatro dimensiones de la socialización (entrenamiento, comprensión funcional de la organización, apoyo de los compañeros de trabajo y perspectivas de futuro), que también consideraban las seis áreas identificadas por Chao et 
al. (1994) (eficiencia de rendimiento, política, lenguaje, personas, objetivos/ valores organizacionales e historia), así como las cinco áreas de socialización del modelo de Cooper-Thomas y Anderson (2006) (tarea, rol y desempeño; compañero de trabajo, social y de grupo; historia, metas y organización; y perspectivas futuras).

Diferentes autores han encontrado poca investigación sobre factores individuales, y que algunos de ellos como la autoeficacia o las necesidades pueden influir recíprocamente en el proceso de socialización organizacional (Fisher, 1986; Jones, 1986; Bauer et al., 1998; Taormina, 2009). Sus evidencias muestran, por una parte, cómo las relaciones de apoyo mutuo y el apoyo recibido de los compañeros durante la socialización, facilita la creación de compromiso con la organización (Meyer, Allen \& Topolnytsky, 1998; Tierney, Bauer \& Potter, 2002; Filstad, 2004; 2011; Mitus, 2006; Calderón Mafud, et al., 2015). Por otro lado, el entrenamiento, el soporte emocional y la comprensión funcional que un trabajador obtiene de sus compañeros actúan para que este pueda enfrentar los cambios organizacionales que lo obligan a clarificar su rol constantemente (Lapointe, Vandenbergbe \& Boudrias, 2014; Feldman, 1981). Además, la socialización organizacional permite que un trabajador perciba tener satisfacción en el trabajo gracias a que sus compañeros le brindan aprendizaje y apoyo para clarificar su rol laboral (Autry \& Daugherty, 2003; Chao et al., 1994; Schmidt, 2010; Taormina, 1997).

Por último, las perspectivas de futuro están relacionadas con la retención de talento en las organizaciones, porque las recompensas extrínsecas entregadas por la organización logran que un empleado desee continuar siendo miembro de la misma (Chen, Ployhart, Thomas, Anderson y Bliese, 2011; Taormina, 1994, 1997, 2004).

En suma, la investigación sobre la socialización organizacional ha encontrado evidencias de que este proceso influye en el compromiso de los trabajadores, mejora su nivel de satisfacción con el empleo, incrementa las relaciones de apoyo y ayuda a clarificar su rol laboral aumentando su nivel de autoeficacia y genera niveles elevados de engagement en poblaciones estudiadas.

\subsection{Work engagement}

El estudio de los estados psicológicos positivos es muy reciente en el ámbito laboral. El estado conocido como engagement involucra a otros factores que facilitan que un empleado pueda desarrollarse con eficacia dentro de una organización. Se define como un estado de energía, vigor, entusiasmo, 
motivación y compromiso relacionados con la productividad; podría decirse que una persona que está en un estado de engagement está física, cognitiva y emocionalmente implicada con su rol en el trabajo (Kahn, 1990; Schaufeli, et al., 2002).

El engagement puede ser concebido también como una interdependencia recíproca entre los intereses económicos de una organización y los aspectos socioemocionales de sus miembros, los cuales al interactuar hacen crecer a todos en conjunto, es decir, que mientras la empresa satisface sus intereses y metas, esta facilita a sus miembros desarrollarse, adquirir aprendizajes que les consientan ascender en su carrera y experimentar bienestar y satisfacción laboral (Saks, 2006; Schaufeli, Taris \& Van Rhenen, 2008).

El estado de engagement está íntimamente ligado a la productividad debido a que les permite a los miembros de las organizaciones aprovechar mejor sus roles laborales y es un complemento emocional para que el trabajador pueda realizar sus tareas de manera eficiente (Kahn, 1990; May, Gilson \& Harter, 2004; Xanthopoulou, Bakker, Demerouti, \& Schaufeli, 2009). Sin embargo, este estado positivo no proviene únicamente de factores personales, sino que depende de algunas características culturales de las organizaciones como la retroalimentación, el fomento a la autonomía, brindar oportunidades de aprendizaje, y opciones para el desarrollo de carrera, lo cual genera un mayor contacto interpersonal, trabajo en equipo, e interés en los compañeros (Konstantellou, 2001).

De acuerdo con esto, se sabe que cuando las empresas fomentan procesos de socialización en los que todos accedan a una culturización uniforme, ello puede generar que el estado de engagement aparezca de manera grupal, en ciertos equipos de trabajo, o incluso partes de la organización (Salanova, Agut \& Peiró, 2005). La socialización organizacional cumple un papel importante en los sistemas que se enfocan a aumentar la productividad de una empresa. Al tomar en cuenta las necesidades de sus miembros, una organización facilita su implicación en la cultura, y estos a su vez, se interesan en tratar de cumplir con los valores y necesidades de la organización (Schaufeli \& Salanova, 2007).

Se han encontrado relaciones entre el work engagement y los procesos de socialización y liderazgo en la organización; se sabe que una persona podría ser influenciada por el apoyo de sus compañeros en el trabajo, sin embargo, 
son los líderes quienes están involucrados directamente con la vida laboral de los individuos, de esa manera fomentan y tienen un papel importante en la determinación del nivel de engagement en los empleados (Bamford, et al., 2013).

Finalmente, como hipótesis principal se plantea que la socialización como proceso relacionado con estados de autoeficacia, la claridad de rol y en general con estados de satisfacción en el trabajo, que permiten que se eleven los niveles de vigor, y que al mostrarse competencias relacionadas con el trabajo y organización, los trabajadores muestren mayor dedicación a las tareas y su absorción. Es decir, los dominios de la socialización organizacional mostrarían efectos significativos en las tres dimensiones del work engagement y para evidenciarlo, se planteó realizar correlaciones de Pearson, además de tres modelos de regresión lineal múltiple paso a paso.

\section{MÉTODO}

\subsection{Diseño}

El diseño que se llevó a cabo fue un estudio transversal-correlacional analítico con la finalidad de establecer efectos de los dominios de la socialización sobre los componentes del work engagement.

\subsection{Instrumentos}

\subsubsection{Inventario de Socialización Organizacional validado al español}

Por Bravo, Gómez-Jacinto y Montalbán (2004), a partir de Taormina (1994). Se compone de 20 ítems divididos en cuatro escalas: entrenamiento, comprensión, apoyo de los compañeros y perspectivas de futuro. Cada reactivo es una afirmación respecto a la forma en que se desarrolla la socialización organizacional del participante, por ejemplo, "la formación recibida me ha ofrecido un completo conocimiento de las habilidades necesarias para mi trabajo" (reactivo 3), "creo conocer muy bien cómo funciona la institución" (reactivo 10), o "puedo prever fácilmente mis posibilidades de ascenso dentro de la institución" (reactivo 18). Se responde mediante una escala Likert de cinco puntos desde 1 "Nunca" hasta 5 "Siempre". Para su calificación se obtienen las medias y desviaciones típicas de los participantes en cada una de las cuatro escalas. En su validación de la traducción española de Bravo, et al. (2004) realizada con una muestra de 370 trabajadores con media de edad 24 años reportaron una confiabilidad, alfa de Cronbach, para el total del cuestionario, de .79 . 


\subsubsection{Utrecht Work Engagement Scale (Schaufeli et al., 2002)}

Elaborada en su versión al español por Schaufeli, Bakker y Salanova (2006). Se compone por tres subescalas de cinco ítems cada una: vigor, dedicación y absorción, que los participantes deben responder en una respuesta tipo Likert de seis puntos desde 0 "Nunca-Ninguna vez" hasta 6 "Siempre-todos los días. Un ejemplo de ítem para vigor es "En mi trabajo me siento lleno de energía", para dedicación "Estoy entusiasmado con mi trabajo" y, por último, "El tiempo 'vuela' cuando estoy trabajando" es un ejemplo de la dimensión absorción. La consistencia interna (alpha de Cronbach) para vigor, dedicación y absorción es de $.83, .89$ y .82 , respectivamente.

\subsection{Participantes}

Participaron en el estudio 187 empleados de cinco organizaciones distintas, pertenecientes a los municipios de Colima y Manzanillo en México. Estos participantes fueron seleccionados aleatoriamente al salir o llegar a sus trabajos y se les entregó un cuestionario solo cuando se trataba de empleados contratados y con al menos un año trabajando. La edad media de la muestra fue el rango de 26 a 30 años. El 37\% tiene más de 3 años de antigüedad en el empleo y un $53 \%$ de la muestra de participantes estuvo formado por mujeres.

\subsection{Procedimiento}

Se seleccionaron organizaciones como unidades de observaciones bajo el siguiente procedimiento: El marco muestral se realizó a partir de la consulta en el Directorio Estadístico Nacional de Unidades Económicas (http://www3. inegi.org.mx) Se seleccionaron áreas geográficas de Colima y Manzanillo. Se obtuvo un marco muestral de 785 empresas.

Se desarrolló un esquema de muestreo probabilístico (que consideró la participación de las variables de personal ocupado total) y estratificado con selección independiente para cada estrato por dominio de estudio. El componente principal es una combinación lineal de las variables personal ocupado total y contribución a su sector. La estratificación del componente mediante el algoritmo de k-medias aplicado al componente principal generó cinco estratos. El cálculo de la muestra resultó en un total de 187 participantes seleccionados de tres estratos de acuerdo con su contribución al universo.

Posterior a la selección de las empresas, se eligieron empleados al azar durante la hora de salida de sus trabajos, en donde se les informó del objetivo del estudio y se les solicitó su colaboración voluntaria, mediante el uso de un 
formato de consentimiento informado que detallaba los objetivos y el procedimiento del estudio, el cual se presentó a cada uno de los participantes de la investigación.

\subsection{Análisis de datos}

Los datos se analizaron mediante correlaciones de Pearson para determinar el nivel de relación entre factores de cada variable, y de forma posterior se hicieron modelos de regresión hacia adelante para cada componente de work engagement con los dominios de la socialización.

\subsection{Conflicto de interés}

Durante la planeación y realización de esta investigación no existió ningún conflicto de interés, con ningún tercero o con los participantes.

\section{RESULTADOS}

En la tabla 1 se muestran las correlaciones $r$ de Pearson entre las dimensiones de socialización organizacional y las características del engagement. Se observó que las dimensiones de comprensión funcional y perspectivas de futuro correlacionan de forma media y positiva $(.422 * *$ y $.450 * *$ respectivamente) con las tres características del engagement. El entrenamiento mostró una baja correlación con el engagement, al igual que el apoyo de los compañeros (.260 y .276 respectivamente), siendo relevante para estos resultados este dato debido a que el funcionamiento secuencial de la socialización inicia por el entrenamiento.

\section{Tabla 1.}

Correlaciones entre las dimensiones de Socialización Organizacional y las características de Engagement

\begin{tabular}{cccccc}
\hline & $\begin{array}{c}\text { SOCIALIZACIÓN } \\
\text { ORGANIZACIONAL }\end{array}$ & ENTRENAMIENTO & COMPRENSIÓN & $\begin{array}{c}\text { APOYO A } \\
\text { COMPAÑEROS }\end{array}$ & $\begin{array}{c}\text { PERSPECTIVAS A } \\
\text { FUTURO }\end{array}$ \\
\hline Engagement & $.420^{* *}$ & $.260^{* *}$ & $.422^{* *}$ & $.276^{* *}$ & $.450^{* *}$ \\
Vigor & $.350^{* *}$ & $.196^{*}$ & $.371^{* *}$ & $.206^{*}$ & $.406^{* *}$ \\
Dedicación & $.469^{* *}$ & $.300^{* *}$ & $.440^{* *}$ & $.334^{* *}$ & $.482^{* *}$ \\
Absorción & $.343^{* *}$ & $.221^{*}$ & $.359^{* *}$ & $.219^{*}$ & $.358^{* *}$ \\
\hline
\end{tabular}

Nota: ${ }^{*} p<.05, * * p<.01$

Para analizar en qué medida las dimensiones de Comprensión y Perspectivas a futuro podrían ejercer efecto sobre los componentes del engagement de los participantes, se crearon tres modelos de regresión múltiple para cada una de las tres dimensiones del work engagement, utilizando los puntajes de cada uno de estos como variable criterio (variable dependiente) y las dimensiones de socialización organizacional como variables predictoras. 
En la Tabla 2 en el modelo para la variable vigor, se mostró una contribución significativa de los dominios Comprensión funcional de la organización (.290) y Perspectivas de Futuro (.211) como antecedentes del vigor.

Tabla 2.

Análisis de regresión múltiple para Vigor

\begin{tabular}{ccccc}
\hline VARIABLE & $\mathbf{B}^{\text {a }}$ & EE $^{\text {b }}$ & BETA $^{\mathbf{c}}$ & $\mathbf{T}^{\text {d }}$ \\
\hline Comprensión & .313 & .113 & .290 & $2.776^{* *}$ \\
Perspectivas a futuro & .353 & .174 & .211 & $2.025^{* *}$ \\
\hline
\end{tabular}

** (Significancia del estadístico t) $p<.05$

a. B=Coeficiente de regresión no estandarizado.

b. $E E=E$ Eror estándar de la estimación

c. $\mathrm{B}=$ Coeficiente de regresión estandarizado

d. $\mathrm{t}=$ Estadístico $\mathrm{t}$.

En el segundo modelo, para el componente dedicación, como se observa en a Tabla 3, también apareció de forma significativa la contribución de los dominios Comprensión funcional de la organización (.344) y Perspectivas de Futuro (.251) para explicar la dedicación.

Tabla 3.

Análisis de regresión múltiple para Dedicación

\begin{tabular}{lcccc}
\hline \multicolumn{1}{c}{ VARIABLE } & B $^{\text {a }}$ & EE $^{\text {b }}$ & BETA $^{\mathbf{c}}$ & T $^{\text {d }}$ \\
\hline \multicolumn{1}{c}{ Comprensión } & .407 & .117 & .344 & $3.474^{* *}$ \\
Perspectivas a futuro & .459 & .181 & .251 & $2.536^{* *}$ \\
\hline$* * \quad$ Significancia del estadístico t) $p<.05$ & & & \\
a. B=Coeficiente de regresión no estandarizado. & & \\
b. EE=Error estándar de la estimación \\
c. B=Coeficiente de regresión estandarizado \\
d. t= Estadístico t.
\end{tabular}

Finalmente, en el modelo para el factor conocido como absorción, nuevamente la contribución de los dominios Comprensión funcional de la organización (.232) y Perspectivas de Futuro (.231) apareció para explicar el funcionamiento de la absorción como efecto.

Tabla 4.

Análisis de regresión múltiple para Absorción

\begin{tabular}{ccccc}
\hline VARIABLE & $\mathbf{B}^{\mathbf{a}}$ & EE $^{\mathrm{b}}$ & BETA $^{\mathbf{c}}$ & T $^{\mathbf{d}}$ \\
\hline Comprensión & .397 & .182 & .232 & $2.181^{* *}$ \\
Perspectivas a futuro & .256 & .118 & .231 & $2.171^{* *}$ \\
\hline
\end{tabular}

** (Significancia del estadístico t) $p<.05$

a. $\mathrm{B}=$ Coeficiente de regresión no estandarizado.

b. $\mathrm{EE}=$ Error estándar de la estimación

c. $B=$ Coeficiente de regresión estandarizado

d. $\mathrm{t}=$ Estadístico $\mathrm{t}$. 
Los resultados muestran que en general, existen correlaciones positivas entre todos los dominios del work engagement y los de la socialización organizacional; mientras que los resultados del análisis de regresión múltiple (Tablas 1 , Tabla 2 y Tabla 3) mostraron que sólo los dominios de comprensión y perspectivas a futuro aparecen como contribuciones sobre las tres características del estado de work engagement.

\section{DISCUSIONES}

Los resultados obtenidos indican que la Socialización organizacional presenta correlación positiva significativa con el Engagement. Lo que indica que el Engagement pude ser resultado de una Socialización adecuada, coincidiendo así con lo encontrado por Lisbona, et al., (2009).

De manera más específica, se encontró que el dominio de entrenamiento tiene relación positiva sobre el engagement debido a que al tener empleados capacitados y con las habilidades o destrezas que le permitan desempeñar su puesto de manera correcta, genera un amento en sus niveles de motivación permitiéndole tener mayor energía, persistencia y deseos por esforzarse al realizar las tareas (Maslach, Schaufeli \& Leiter, 2001; Borrego, 2016). Se enfatiza el hecho de que, aunque la correlación es baja, el entrenamiento o formación son la base de la socialización y se sabe que un programa de capacitación continua genera que la autoeficacia en los miembros de la organización aumente (Lisbona, et al., 2009).

Igualmente, aunque los resultados muestran una correlación baja entre el apoyo a compañeros y el engagement, este dominio de la socialización tiene efectos importantes, de forma similar a lo mencionado por Konstantellou (2001) al observar que una de las características principales en empleados engaged es el interés por sus compañeros y el trabajo en equipo. Se considera que el apoyo recibido por parte de miembros de una organización provee al personal de vínculos afectivos que le permiten compartir intereses, metas personales y recursos profesionales con otros, disminuyendo los niveles de despersonalización, típicos en trabajadores que padecen Burnout (Maslach, 2001).

La comprensión funcional de la organización presenta una relación positiva e influencia sobre el engagement, de la misma forma que evidenciaron Maslach, Jackson, Leiter, Schaufeli y Schwab (1986), mencionando que al comprender de manera adecuada su rol laboral, los empleados se perciben más comprometidos con metas y objetivos de la organización; esto les permite 
por otra parte, trazar metas propias que generan satisfacción y motivación para búsqueda de nuevas metas (De Dreu, 2007; Decker, Calo \& Weer, 2012).

Las perspectivas de futuro poseen, al igual que la comprensión, gran influencia sobre la presencia del engagement en los empleados. Si el esfuerzo del personal es recompensado, la motivación se verá en aumento, por consecuencia el vigor y la dedicación por realizar las tareas de la mejor manera, sin importar el tiempo que estas requieran, aumentará en la misma medida (Hakanen, Perhoniemi \& Toppinen-Tanner, 2008).

Los resultados obtenidos nos permiten proponer que el implementar programas que puedan mantener un adecuado nivel de socialización organizacional facilitará la aparición de empleados engaged, los cuales estarán comprometidos y satisfechos con la organización, aumentando así la productividad y el desarrollo de talento. Es importante mencionar que el engagement no es propio de un solo sujeto, si no que puede surgir de manera grupal teniendo impacto en una o varias áreas de la empresa (Salanova, et al., 2005).

Los elementos de la socialización organizacional conocidos como comprensión funcional (entender las normas de la organización y la forma de interactuar en ella) y las perspectivas de futuro (identificar posibilidades de obtener beneficios en el futuro) se combinan para mostrar efectos en el vigor, esto sugiere que posiblemente, la disminución de la ansiedad e incertidumbre obtenida al comprender el ambiente de la organización (Louis, 1980; Feldman \& Brett, 1983) y visualizar posibilidades de ascensos en ella, generan emociones positivas (Fredrickson, 2001) en los empleados, de tal manera que se incrementa la sensación de energía respecto a las tareas específicas, produciendo vigor en el empleado.

Esto es consistente con lo reportado por Macsinga, Sulea, Sârbescu, Fischmann \& Dumitru (2015), quienes estudiaron cómo el empoderamiento psicológico tiene un rol relevante en el desarrollo del work engagement; es probable que al elevarse los niveles de comprensión, también sea posible que los empleados se sientan útiles al interactuar con sus compañeros de trabajo, tratando de pasar más tiempo enfocándose en sus tareas y en desarrollar logros.

La misma combinación de dominios de la socialización (comprender cómo funciona la organización y tener perspectivas de futuro) resultó ser la que 
mejores efectos tuvo en la dedicación a la tarea. Es probable, que también, al entender mejor las normas relativas al rol, la calidad y la gestión de la empresa, además de tener la certidumbre de estabilidad y crecimiento, una persona pueda enfocarse más hacia las tareas propias de su rol, en lugar de tener incertidumbre por aspectos organizativos, normativos y de gestión.

Los efectos de la socialización son para muchos autores producto del apoyo organizacional, más que de la comprensión y las perspectivas de crecimiento en la organización, para ellos la participación en el trabajo está relacionada con la socialización, el apoyo y la dedicación (Bano, Vyas \& Gupta, 2015; Gokul, Sridevi \& Srinivasan, 2012; Rhoades, Eisenberger \& Armeli, 2001)

En complemento a otros autores que encontraron relaciones del work engagement con los recursos del trabajo y las intervenciones de job crafting (Van Wingerden, Derks \& Bakker, 2017), nuestros resultados apuntan a que si bien el work engagement puede ser fomentado a través de mejorar los recursos personales, son las perspectivas de futuro y entender cómo funciona la organización y su estructura de valores, lo que posibilita que una persona consiga incrementar el nivel de absorción en el trabajo.

Finalmente, en el modelo para explicar los antecedentes de la absorción, nuevamente la contribución de los dominios comprensión funcional de la organización y perspectivas de futuro se combinaron para generar efectos en ella. Esta evidencia muestra cómo también este dominio del work engagement parece estar relacionado con los efectos cognitivos de entender la cultura de la organización, como ha mencionado Louis (1980), quien sugería que los individuos buscan crear un sentido a su experiencia en la organización, aseverando que la búsqueda de información reduce la incertidumbre y la ansiedad de los recién llegados; y más recientemente, Saks y Gruman (2018), quienes proponen la necesidad de un modelo de recursos de socialización que solucione el problema de la adaptación constante a los cambios culturales que vive una organización.

Esto es más consistente con lo encontrado con Den, Chen, Yang y Xu (2017), quienes observaron relaciones del work engagement con el éxito que las personas perciben tener en su carrera y cuando son motivadas para invertir tiempo en su trabajo y esto les permite desarrollarse profesionalmente usando los recursos sociales para mejorar su desempeño y el de sus equipos de trabajo (Mäkikangas, Aunola, Seppälä \& Hakanen, 2016). 
Como conclusión es posible establecer argumentos sobre las relaciones encontradas, los complementos a otros hallazgos y las líneas de investigación a seguir. En primer lugar, las correlaciones subrayan que, al socializarse apropiadamente, los empleados pueden elevar su nivel de engagement generando relaciones productivas en el trabajo; sin embargo, los efectos más significativos en esta población no provienen de las relaciones con los compañeros o el entrenamiento, sino de la posibilidad de entender las normas en el trabajo y encontrar estabilidad y crecimiento. Posiblemente esto se deba a las condiciones propias del país y de la región, en donde la inseguridad laboral y los bajos salarios son cotidianos. Entender que, al socializar en organizaciones pequeñas y sencillas, o grandes y complejas construye recursos sociales para las organizaciones y se convierte en sí mismo en un proceso fundamental para facilitar la adaptación, brindar claridad y aportar estabilidad. Por otra parte, es necesario investigar más y de forma experimental en formas específicas de intervención basadas en la formación, el capital psicológico, programas de desarrollo interno y entrenamiento en colaboración a equipos para establecer estos como los recursos principales que utiliza la organización de forma sistemática para generar work engagement en sus trabajadores.

Agradecimientos: A PIENSO en Latinoamérica por la gestión y el apoyo para el levantamiento de información, análisis de datos y equipo de investigadores.

Financiamiento: Esta investigación se financió con recursos propios.

\section{REFERENCIAS}

Allee, V. (1997). The knowledge evolution: Expanding organizational intelligence. Routledge.

Amidon, D. M. (1997). Innovation strategy for the knowledge economy: the Ken Awakening. United Kingdom: Routledge.

Autry, C. W., \& Daugherty, P. J. (2003). Warehouse operations employees: linking person-organization fit, job satisfaction, and coping responses. Journal of Business Logistics, 24(1), 171-197. https://doi.org/10.1002/j.2158-1592.2003.tb00036.x

Bamford, M., Wong, C. A., \& Laschinger, H. (2013). The influence of authentic leadership and areas of worklife on work engagement of registered nurses. Journal of nursing management, 21(3), 529-540. https://doi.org/10.1111/j.13652834.2012.01399.x

Bano, S., Vyas, K., \& Gupta, R. (2015). Perceived Organisational Support and Work Engagement: A Cross Generational Study. Journal of Psychosocial Research, 10(2), 357-364. https://search.proquest.com/openview/4a4f5b4e5a318c2537d429f3abe8fe43/1 ?pq-origsite $=$ gscholar $\&$ cbl=506336

Bauer, T., Morrison, M., Callister, Y., \& Ferris, T. (1998). Organizational socialization: A review and directions for future research. Research in Personnel and Human Resources Management, 16, 149-214. https://www.researchgate.net/profile/ 
Talya_Bauer/publication/44019010_Socialization_Research_A_Review_and_ Directions_for_Future_Research/links/54625be20cf2cb7e9da64d69/Socialization-Research-A-Review-and-Directions-for-Future-Research.pdf

Bauer, T. N., \& Erdogan, B. (2014). Delineating and reviewing the role of newcomer capital in organizational socialization. Annu. Rev. Organ. Psychol. Organ. Behav., 1(1), 439-457. https://doi:10.1146/annurev-orgpsych-031413-091251

Bauer, T. N., Bodner, T., Erdogan, B., Truxillo, D. M., \& Tucker, J. S. (2007). Newcomer adjustment during organizational socialization: a meta-analytic review of antecedents, outcomes, and methods. Journal of Applied Psychology, 92(3), 707. https:// pdxscholar.library.pdx.edu/cgi/viewcontent.cgi?referer=https://scholar.google. com/\&httpsredir=1\&article $=1026 \&$ context=busadmin_fac

Borrego, Y. (2016). El Engagement en el trabajo: Antecedentes y resultados organizacionales. (Doctoral dissertation Tesis doctoral). Universidad de Huelva, Huelva. http://hdl.handle.net/10272/11948

Bravo, M., Gómez-Jacinto, T., \& Montalbán, S. (2004). Inventario de Socialización Organizacional validado al español. http://revistas.ibero.mx/psicologia/articulo_ detalle.php?pageNum_paginas=2\&totalRows_paginas $=10 \& i d$ _volumen $=11 \& i d$ _ articulo $=117$ \&pagina $=1 \&$ pagina $=2$

Cable, D. M., \& Parsons, C. K. (2001). Socialization tactics and person-organization fit. Personnel Psychology, 54(1), 1-23. https://doi.org/10.1111/j.1744-6570.2001. tb00083.x

Calderón Mafud, J. L., Laca Arocena, F. A., Pando Moreno, M., \& Pedroza Cabrera, F. J. (2015). Relationship between Mexican Workers' Organizational Socialization and Commitment. Psicogente, 18(34), 267-277. http://doi.org/10.17081/ psico.18.34.503

Chao, G. T., O'Leary-Kelly, A. M., Wolf, S., Klein, H. J., \& Gardner, P. D. (1994). Organizational socialization: Its content and consequences. Journal of Applied Psychology, 79(5), 730. https://www.researchgate.net/profile/Howard_Klein2/ publication/232462733_Organizational_Socialization_Its_Content_and_Consequences/links/0c96052d061d8460a9000000.pdf

Chen, G., Ployhart, R. E., Thomas, H. C., Anderson, N., \& Bliese, P. D. (2011). The power of momentum: A new model of dynamic relationships between job satisfaction change and turnover intentions. Academy of Management Journal, 54(1), 159-181. https://doi.org/10.5465/amj.2011.59215089

Cooper-Thomas, H. D., \& Anderson, N. (2006). Organizational socialization: A new theoretical model and recommendations for future research and HRM practices in organizations. Journal of Managerial Psychology, 21(5), 492-516. https://doi. org/10.1108/02683940610673997

Csikszentmihalyi, M. (1990). Flow: The psychology of optimal performance. NY: Cambridge UniversityPress.

Danielson, M. M. (2004). A theory of continuous socialization for organizational renewal. Human Resource Development Review, 3(4), 354-384. https://doi. org/10.1177\%2F1534484304271528

De Dreu, C. K. (2007). Cooperative outcome interdependence, task reflexivity, and team effectiveness: a motivated information processing perspective. Journal of applied psychology, 92(3), 628. https://doi.org/10.1037/0021-9010.92.3.628

Decker, W. H., Calo, T. J., \& Weer, C. H. (2012). Affiliation motivation and interest in entrepreneurial careers. Journal of Managerial Psychology, 27(3), 302-320. https://doi.org/10.1108/02683941211205835 
Den, J., Chen, Q., Yang, S., \& Xu, Y. (2017). Effect of Leaders' Work Engagement on Followers' Subjective Career Success: A Multi-Level Model. International Management Review, 13(2), 45-52. http://scholarspress.us/conferences/pdf/ Health-Wuhan.pdf\#page $=40$

Denison, D. R., \& Mishra, A. K. (1995). Toward a theory of organizational culture and effectiveness. Organization Science, 6(2), 204-223. https://doi.org/10.1287/ orsc.6.2.204

Ellis, A. M., Bauer, T. N., Mansfield, L. R., Erdogan, B., Truxillo, D. M., \& Simon, L. S. (2015). Navigating uncharted waters: Newcomer socialization through the lens of stress theory. Journal of Management, 41(1), 203-235. https://doi. org/10.1177\%2F0149206314557525

Feldman, D. C. (1981). The multiple socialization of organization members. Academy of Management Review, 6(2), 309-318. https://doi.org/10.5465/amr.1981.4287859

Feldman, D. C., \& Brett, J. M. (1983). Coping with new jobs: A comparative study of new hires and job changers. Academy of Management Journal, 26(2), 258-272. https://doi.org/10.5465/255974

Filstad, C. (2004). How newcomers use role models in organizational socialization. Journal of Workplace Learning, 16(7), 396-409. https://doi. org/10.1108/13665620410558297

Filstad, C. (2011). Organizational commitment through organizational socialization tactics. Journal of Workplace Learning, 23(6), 376-390. https://doi. org/10.1108/13665621111154395

Fisher, C. D. (1986). Organizational socialization: An integrative review. Research in Personnel and Human Resources Management, 4(1), 101-145. http://faculty. babson.edu/krollag/org_site/org_theory/socialization_notes/fisher_integ.html

Fredrickson, B. L. (2001). The role of positive emotions in positive psychology: The broaden-and-build theory of positive emotions. American Psychologist, 56(3), 218. http://psycnet.apa.org/doi/10.1037/0003-066X.56.3.218

Gokul, A., Sridevi, G., \& Srinivasan, P. T. (2012). The relationship between perceived organizational support, work engagement and affective commitment. AMET International Journal of Management, Vol 5. 29-37. http://www.ametjournal. com/attachment/ametjournal-4/Dev-Article-4-Gokul.pdf

Gregory, B. T., Harris, S. G., Armenakis, A. A., \& Shook, C. L. (2009). Organizational culture and effectiveness: A study of values, attitudes, and organizational outcomes. Journal of Business Research, 62(7), 673-679. https://doi. org/10.1016/j.jbusres.2008.05.021

Hakanen, J. J., Perhoniemi, R., \& Toppinen-Tanner, S. (2008). Positive gain spirals at work: From job resources to work engagement, personal initiative and work-unit innovativeness. Journal of Vocational Behavior, 73(1), 78-91. https://doi. org/10.1016/j.jvb.2008.01.003

Hesketh, B., \& Myors, B. (1997). How should we measure fit in organisational psychology-or should we? Australian Psychologist, 32(1), 71-76. https://researchers. mq.edu.au/en/publications/how-should-we-measure-fit-in-organisational-psychology-or-should-

Holton, E. F. (1996). The flawed four-level evaluation model. Human Resource Development Quarterly, 7(1), 5-21. https://doi.org/10.1002/hrdq.3920070103

Jones, G. R. (1986). Socialization tactics, self-efficacy, and newcomers' adjustments to organizations. Academy of Management Journal, 29(2), 262-279. https://doi. org/10.5465/256188 
Kahn, W. A. (1990). Psychological conditions of personal engagement and disengagement at work. Academy of Management Journal, 33(4), 692-724. https://doi. org/10.5465/256287

Klein, H. J., \& Weaver, N. A. (2000). The effectiveness of an organizational-level orientation training program in the socialization of new hires. Personnel Psychology, 53(1), 47-66. https://doi.org/10.1111/j.1744-6570.2000.tb00193.x

Konstantellou, E. (2001). An Exploratory Investigation of Enjoyment at Work: A Cross-National Approach. (Unpublished Master Thesis). UK, University of Surrey.

Lapointe, É., Vandenberghe, C., \& Boudrias, J. S. (2014). Organizational socialization tactics and newcomer adjustment: The mediating role of role clarity and affectbased trust relationships. Journal of Occupational and Organizational Psychology, 87(3), 599-624. https://doi.org/10.1111/joop.12065

Lisbona, A., Morales, J., \& Palací, F. J. (2009). El engagement como resultado de la socialización organizacional. International Journal of Psychology and Psychological Therapy, 9(1). https://dialnet.unirioja.es/servlet/articulo?codigo=2873396

Louis, M. R. (1980). Surprise and sense making: What newcomers experience in entering unfamiliar organizational settings. Administrative Science Quarterly, 4(1), 226-251. https://doi.org/10.1300/J111V04N01_08

Lund, D. B. (2003). Organizational culture and job satisfaction. Journal of Business \& IndustrialMarketing,18(3),219-236.https://doi.org/10.1108/0885862031047313

Luthans, F., Norman, S. M., Avolio, B. J., \& Avey, J. B. (2008). The mediating role of psychological capital in the supportive organizational climate-employee performance relationship. Journal of Organizational Behavior: The International Journal of Industrial, Occupational and Organizational Psychology and Behavior, 29(2), 219-238. https://doi.org/10.1002/job.507

Macintosh, E. W., \& Doherty, A. (2010). The influence of organizational culture on job satisfaction and intention to leave. Sport Management Review, 13(2), 106-117. https://doi.org/10.1016/j.smr.2009.04.006

Macsinga, I., Sulea, C., Sârbescu, P., Fischmann, G., \& Dumitru, C. (2015). Engaged, Committed and Helpful Employees: The Role of Psychological Empowerment. Journal of Psychology, 149(3), 263-276. https://doi.org/10.1080/00223980.201 3.874323

Mafud, J., Arocena, F., \& Moreno, M. (2017). La autoeficacia como mediador entre el estrés laboral y el bienestar. Psicología y Salud, 27(1), 71-78. http://psicologiaysalud.uv.mx/index.php/psicysalud/article/view/2438/4291

Major, D. A., Kozlowski, S. W., Chao, G. T., \& Gardner, P. D. (1995). A longitudinal investigation of newcomer expectations, early socialization outcomes, and the moderating effects of role development factors. Journal of Applied Psychology, 80(3), 418. http://psycnet.apa.org/doi/10.1037/0021-9010.80.3.418

Mäkikangas, A., Aunola, K., Seppälä, P., \& Hakanen, J. (2016). Work engagement-team performance relationship: shared job crafting as a moderator. Journal of Occupational \& Organizational Psychology, 89(4), 772-790. https://doi.org/10.1111/ joop.12154

Maslach, C. (2001). What have we learned about burnout and health? Psychology \& Health, 16(5), 607-611. https://doi.org/10.1080/08870440108405530

Maslach, C., Jackson, S. E., Leiter, M. P., Schaufeli, W. B., \& Schwab, R. L. (1986). Maslach burnout inventory (Vol. 21), Palo Alto, CA: Consulting Psychologists Press.

Maslach, C., Schaufeli, W. B., \& Leiter, M. P. (2001). Job burnout. Annual Review of Psychology, 52(1), 397-422. https://doi.org/10.1146/annurev.psych.52.1.397 
May, D. R., Gilson, R. L., \& Harter, L. M. (2004). The psychological conditions of meaningfulness, safety and availability and the engagement of the human spirit at work. Journal of Occupational and Organizational Psychology, 77(1), 11-37. https://doi.org/10.1348/096317904322915892

McKinnon, J. L., Harrison, G. L., Chow, C. W., \& Wu, A. (2003). Organizational culture: Association with commitment, job satisfaction, propensity to remain, and information sharing in Taiwan. International Journal of Business Studies, 11(1), 25. https://www.researchgate.net/profile/Chee_Chow/ publication/266469757_Organizational_Culture_Association_with_ Commitment_Job_Satisfaction_Propensity_to_Remain_and_Information_ Sharing_in_Taiwan/links/54b3dcfa0cf26833efcfd83c/Organizational-Culture-Association-with-Commitment-Job-Satisfaction-Propensity-to-Remain-and-Information-Sharing-in-Taiwan.pdf

Meyer, J. P., Allen, N. J., \& Topolnytsky, L. (1998). Commitment in a changing world of work. Canadian Psychology/Psychologie Canadienne, 39(1-2), 83. http://psycnet. apa.org/doi/10.1037/h0086797

Mitus, J. S. (2006). Organizational socialization from a content perspective and its effect on the affective commitment of newly hired rehabilitation counselors. Journal of Rehabilitation, 72(2), 12. https://www.researchgate.net/publication/288468490_Organizational_socialization_from_a_content_perspective_ and_its_effect_on_the_affective_commitment_of_newly_hired_rehabilitation_ counselors

Nelson, D. L., \& Quick, J. C. (1991). Social support and newcomer adjustment in organizations: Attachment theory at work? Journal of Organizational Behavior, 12(6), 543-554. https://doi.org/10.1002/job.4030120607

Nonaka, L., Takeuchi, H., \& Umemoto, K. (1996). A theory of organizational knowledge creation. International Journal of Technology Management, 11(7-8), 833-845. https://doi.org/10.1504/IJTM.1996.025472

Rhoades, L., Eisenberger, R., \& Armeli, S. (2001). Affective commitment to the organization: The contribution of perceived organizational support. Journal of Applied Psychology, 86(5), 825. https://s3.amazonaws.com/academia.edu. documents/37090660/Affective_commitment_to_the_organization_The_contribution_of_perceived_organizational_support.pdf?AWSAccessKeyld=AKIAIWOWYYGZ2Y53UL3A\&Expires=1535937344\&Signature=Z3AU\%2BINT3KjLTdALBTkY18afdwY\%3D\&response-content-disposition=inline\%3B\%20filename\%3DAffective_commitment_to_the_organization.pdf

Rollag, K., Parise, S., \& Cross, R. (2005). Getting new hires up to speed quickly. MIT Sloan Management Review, 46(2), 35. https://dialnet.unirioja.es/servlet/articulo?codigo $=2169682$

Saks, A. M. (1996). The Relationship Between the Amount and Helpfulness of Entry Thaining and Work Outcomes. Human Relations, 49(4), 429-451. https://doi. org/10.1177\%2F001872679604900402

Saks, A. M. (2006). Antecedents and consequences of employee engagement. Journal of Managerial Psychology, 21(7), 600-619. https://doi. org/10.1108/02683940610690169

Saks, A. M., \& Gruman, J. A. (2018). Socialization resources theory and newcomers' work engagement: a new pathway to newcomer socialization. Career Development International, 23(1), 12-32. https://doi.org/10.1108/CDI-12-2016-0214

Salanova, M., Agut, S., \& Peiró, J. M. (2005). Linking organizational resources and work engagement to employee performance and customer loyalty: the mediation 
of service climate. Journal of Applied Psychology, 90(6), 1217. http://psycnet.apa. org/doi/10.1037/0021-9010.90.6.1217

San Park, J., \& Hyun Kim, T. (2009). Do types of organizational culture matter in nurse job satisfaction and turnover intention? Leadership in Health Services, 22(1), 20-38. https://doi.org/10.1108/17511870910928001

Schaufeli, W. B., Bakker, A. B., \& Salanova, M. (2006). The measurement of work engagement with a short questionnaire: A cross-national study. Educational and Psychological Measurement, 66(4), 701-716. https://doi. org/10.1177\%2F0013164405282471

Schaufeli, W. B., Salanova, M., González-Romá, V., \& Bakker, A. B. (2002). The measurement of engagement and burnout: A two sample confirmatory factor analytic approach. Journal of Happiness Studies, 3(1), 71-92. https://www.wilmarschaufeli.nl/publications/Schaufeli/178.pdf

Schaufeli, W. B., Taris, T. W., \& Van Rhenen, W. (2008). Workaholism, burnout, and work engagement: three of a kind or three different kinds of employee wellbeing? Applied Psychology, 57(2), 173-203. https://doi.org/10.1111/j.14640597.2007.00285.x

Schaufeli, W., \& Salanova, M. (2007). Work engagement. Managing Social and Ethical Issues in Organizations, 135, 177. https://s3.amazonaws.com/academia.edu. documents/8418176/608_eng.pdf?AWSAccessKeyld=AKIAIWOWYYGZ2Y53UL3A\&Expires $=1535937548 \&$ Signature $=028 y B z N y N e 6 d Q q M \% 2 F O I \% 2 B 7 e 950-$ t04\%3D\&response-content-disposition=inline\%3B\%20filename\%3DWork_ engagement.pdf

Schein, E. H. (1996). Culture: The missing concept in organization studies. Administrative Science Quarterly, 41(2), 229-240. https://doi.org/10.2307/2393715

Schmidt, S. W. (2010). The Relationship between Job Training and Job Satisfaction: A Review of Literature. International Journal of Adult Vocational Education and Technology (ljavet), 1(2), 19-28. https://doi.org/10.4018/javet.2010040103

Silverthorne, C. (2004). The impact of organizational culture and person-organization fit on organizational commitment and job satisfaction in Taiwan. Leadership \& Organization Development Journal, 25(7), 592-599. https://doi. org/10.1108/01437730410561477

Simons, J. C., \& Buitendach, J. H. (2013). Psychological capital, work engagement and organisational commitment amongst call centre employees in South Africa. SA Journal of Industrial Psychology, 39(2), 1-12. http://dx.doi.org/10.4102/sajip. v39i2.1071

Song, Z., Chon, K., Ding, G., \& Gu, C. (2015). Impact of organizational socialization tactics on newcomer job satisfaction and engagement: Core self-evaluations as moderators. International Journal of Hospitality Management, 46, 180-189. https://doi.org/10.1016/j.ijhm.2015.02.006

Taormina, G. R., \& Gao, J. (2008). Effects of Organizational Socialization on Work Enthusiasm in Two Chinese Cultures. In 65th Annual Meeting of the Academy of Management, Honolulu, USA. https://doi.org/10.1108/15587890880000405

Taormina, R. J. (1994). The organizational socialization inventory. International journal of Selection and Assessment, 2(3), 133-145. http://umir.umac.mo/ jspui/bitstream/123456789/15713/1/10492_Taormina\%20\%26\%20Gao\%20 (2005)\%20Effects\%20of\%20Organizational\%20Socialization\%20on\%20Work\%20 Enthusiasm.pdf

Taormina, R. J. (1997). Organizational socialization: A multidomain, continuous process model. International Journal of Selection and Assessment, 5(1), 29-47. https://doi.org/10.1111/1468-2389.00043 
Taormina, R. J. (2004). Convergent validation of two measures of organizational socialization. The International Journal of Human Resource Management, 15(1), 76-94. https://doi.org/10.1080/0958519032000157357

Taormina, R. J. (2009). Organizational socialization: the missing link between employee needs and organizational culture. Journal of Managerial Psychology, 24(7), 650-676. https://doi.org/10.1108/02683940910989039

Tierney, P., Bauer, T. N., \& Potter, R. E. (2002). Extra-Role Behavior among Mexican Employees: The Impact of LMX, Group Acceptance, and Job Attitudes. International Journal of Selection and Assessment, 10(4), 292-303. https://doi. org/10.1111/1468-2389.00219

Tsai, Y. (2011). Relationship between organizational culture, leadership behavior and job satisfaction. BMC Health Services Research, 11(1), 98. https://doi. org/10.1186/1472-6963-11-98

Van Wingerden, J., Derks, D., \& Bakker, A. B. (2017). The impact of personal resources and job crafting interventions on work engagement and performance. Human Resource Management, 56(1), 51-67. https://doi.org/10.1002/hrm.21758

Vandenberg, R. J., \& Scarpello, V. (1994). A longitudinal assessment of the determinant relationship between employee commitments to the occupation and the organization. Journal of Organizational Behavior, 15(6), 535-547. https://doi. org/10.1002/job.4030150605

Wallach, E. (1983). Individuals and organization: the cultural match. Training and Development Journal, 12, 28-36. https://psycnet.apa.org/record/1983-22213001

Wang, M., Kammeyer-Mueller, J., Liu, Y., \& Li, Y. (2015). Context, socialization, and newcomer earning. Organizational Psychology Review, 5(1), 3-25. https://doi. org/10.1177\%2F2041386614528832

Wanous, J. P. (1980). The Entry of Newcomers into Organizations. Michigan State Univ East Lansing Dept of Psychology. http://www.dtic.mil/dtic/tr/fulltext/u2/ a091383.pdf

Wanous, J. P. (1992). Organizational entry: Recruitment, selection, orientation, and socialization of newcomers. Upper Saddle River, N. J.: Prentice Hall.

Xanthopoulou, D., Bakker, A. B., Demerouti, E., \& Schaufeli, W. B. (2009). Work engagement and financial returns: A diary study on the role of job and personal resources. Journal of Occupational and Organizational Psychology, 82(1), 183-200. https://doi.org/10.1348/096317908X285633

\section{(cc) BY}


